Journal for ImmunoTherapy of Cancer \section{agonist therapy to incite durable protective antitumor immunity \\ Overcoming resistance to STING}

To cite: Lemos H, Ou R, McCardle C, et al. Overcoming resistance to STING agonist therapy to incite durable protective antitumor immunity. Journal for ImmunoTherapy of Cancer 2020;8: $\mathrm{e} 001182$. doi:10.1136/jitc-2020-001182

- Additional material is published online only. To view, please visit the journal online (http://dx.doi.org/10.1136/jitc2020-001182).

Accepted 23 July 2020

Check for updates

C) Author(s) (or their employer(s)) 2020. Re-use permitted under CC BY. Published by BMJ.

${ }^{1}$ Translational and Clinical Research Institute, Faculty of Medical Sciences, Newcastle University, Newcastle upon Tyne, UK

${ }^{2}$ Georgia Cancer Center, Augusta University Medical College of Georgia, Augusta, Georgia, USA

Correspondence to Dr Andrew L Mellor; andrew.mellor@ncl.ac.uk

Dr Lei Huang; lei.huang@ncl.ac.uk

Henrique Lemos, ${ }^{1}$ Rong Ou, ${ }^{1}$ Caroline McCardle, ${ }^{1}$ Yijun Lin, ${ }^{1}$ Jessica Calver (D),${ }^{1}$ Jack Minett, ${ }^{1}$ Ahmed Chadli, ${ }^{2}$ Lei Huang (D) , ${ }^{1}$ Andrew L Mellor (D) ${ }^{1}$

\begin{abstract}
Background Activating the Stimulator of Interferon Genes (STING) adaptor incites antitumor immunity against immunogenic tumors in mice, prompting clinical trials to test STING activators. However, STING signaling in the tumor microenvironment (TME) during development of Lewis lung carcinoma (LLC) suppresses antitumor immunity to promote tumor growth. We hypothesized that local immune balance favoring suppression of antitumor immunity also attenuates antitumor responses following STING activation. The purpose of this study was to evaluate how STING activation impacts antitumor responses in mice bearing LLC tumors.

Methods Mice bearing established LLC tumors were treated with synthetic cyclic diadenyl monophosphate (CDA) to activate STING. Mice were monitored to assess LLC tumor growth, survival and protective antitumor immunity. Transcriptional and metabolic analyses were used to identify pathways responsive to CDA, and mice were co-treated with CDA and drugs that disrupt these pathways.
\end{abstract}

Results CDA slowed LLC tumor growth but most CDAtreated mice $(77 \%)$ succumbed to tumor growth. No evidence of tumor relapse was found in surviving CDAtreated mice at experimental end points but mice were not immune to LLC challenge. CDA induced rapid increase in immune regulatory pathways involving programmed death-1 (PD-1), indoleamine 2,3 dioxygenase (ID0) and cyclooxygenase-2 (COX2) in the TME. PD-1 blockade enhanced antitumor responses to CDA and increased mouse survival but mice did not eliminate primary tumor burdens. Two IDO inhibitor drugs had little or no beneficial effects on antitumor responses to CDA. A third IDO inhibitor drug synergized with CDA to enhance tumor control and survival but mice did not eliminate primary tumor burdens. In contrast, co-treatments with CDA and the COX2-selective inhibitor celecoxib controlled tumor growth, leading to uniform survival without relapse, and mice acquired resistance to LLC re-challenge and growth of distal tumors not exposed directly to CDA. Thus, mice co-treated with CDA and celecoxib acquired stable and systemic antitumor immunity.

Conclusions STING activation incites potent antitumor responses and boosts local immune regulation to attenuate antitumor responses. Blocking STING-responsive regulatory pathways synergizes with CDA to enhance antitumor responses, particularly COX2 inhibition. Thus, therapy-induced resistance to STING may necessitate co- treatments to disrupt regulatory pathways responsive to STING in patients with cancer.

\section{INTRODUCTION}

During tumor development local inflammation establishes immune checkpoints (ICPs) that suppress antitumor immunity in primary lesions and tumor-draining lymph nodes (TDLNs), which together constitute the immune tumor microenvironment (TME). Blocking cytotoxic T-lymphocyte-associated protein-4 (CTLA-4) and programmed cell death-1 (PD-1) interactions that contribute to ICPs generated promising clinical responses in some cancers. ${ }^{1}$ PD-1 blockade is effective in up to $45 \%$ of patients with melanoma but is far less effective in other cancers, with $\sim 20 \%$ response rates typical in patients with non-small cell lung carcinoma (NSCLC). ${ }^{2} 3$ Many factors may contribute to differential responses to ICP blockade including heterogeneity in patient genetics, immune status and treatment history, plus variations in the inflammatory and immunological landscapes of specific cancer types. In mouse tumor models, tumor immunogenicity, reflecting differential immune cell infiltration in the TME, correlates with responsiveness to PD-1 blockade. ${ }^{4}$ While immunogenic (hot) tumors such as B16 melanomas transfected to express neo-antigens are responsive to PD-1 blockade, weakly immunogenic (cold) Lewis lung carcinoma (LLC) tumors are refractory to PD-1 blockade. ${ }^{2-5}$ Thus, the LLC model recapitulates the poor clinical responses to PD-1 blockade observed in most patients with lung cancer.

Resistance to PD-1 blockade (nivolumab) therapy in patients with advanced melanoma and renal cell carcinoma correlated with elevated oxidative tryptophan metabolism mediated by indoleamine 2,3 dioxygenase (IDO) following PD-1 blockade, suggesting that adaptive therapy resistance may be a 
barrier to inciting effective and durable clinical responses to immunotherapy. ${ }^{6}$ Poor patient survival after radiotherapy/chemotherapy (RT/CT) also correlated with elevated levels of systemic IDO activity, ${ }^{78}$ implicating IDO as a potential barrier to durable antitumor responses after RT/CT. However, monotherapy to inhibit IDO is ineffective in most patients with cancer and the IDO inhibitor epacadostat did not improve survival of patient with melanoma when combined with PD-1 blockade, raising questions about the role of IDO in resistance to therapy and the efficacy of IDO inhibitor drugs in patients with cancer. $^{9}{ }^{10}$ Nevertheless, regulatory pathways induced during tumor development (intrinsic) and/or responsive to therapy (adaptive) may mediate and potentiate resistance to therapy, respectively. Intrinsic and adaptive therapy resistance may be influenced by tumor type, size and immunogenicity, and patient immune status. Regulatory pathway heterogeneity and redundancy may also impact the potency and plasticity of ICPs before and after therapy.

Direct introduction of innate immune adjuvants into the TME is an alternative strategy to boost antitumor immunity. Intratumoral treatment with a synthetic cyclic diadenyl monophosphate (CDA) derivative to activate the signaling adaptor Stimulator of Interferon Genes (STING) led to long-term tumor-free survival of mice with relatively immunogenic tumors such as B16 melanoma. ${ }^{11}$ CDA treatment induced dendritic cells (DCs) to release interferon type I (IFN-I) and cross-present antigens to activate effector CD8 T cells. Previously, we reported that STING/IFN-I signaling promoted LLC tumor growth due to increased local immune regulation mediated by TDLN DCs expressing IDO. ${ }^{12}$ In the current study, we tested if direct CDA treatment controlled growth of LLC tumors. CDA treatment slowed growth of established LLC tumors and prolonged survival, but most mice still succumbed to primary tumor growth and CDA treatment did not induce surviving mice to acquire stable protective antitumor immunity. Direct CDA treatment stimulated rapid elevation of multiple immune regulatory pathways involving PD-1, IDO and COX2 in the TME. Disrupting each pathway independently enhanced antitumor responses to CDA, particularly co-treatment with a COX2 inhibitor drug. Thus, the intrinsic plasticity of physiological responses to immunotherapy means that monotherapies to disrupt ICPs or activate STING are relatively ineffective, while co-treatments that activate STING and disrupt pathways contributing to ICPs boost antitumor responses by reducing intrinsic and adaptive resistance to STING agonists.

\section{RESULTS}

Established LLC tumors are resistant to the antitumor effects of direct STING activation

B6 mice were inoculated with LLC tumor cells and when dermal tumors were well-established $\left(250-300 \mathrm{~mm}^{3}\right)$ mice were given a short course of direct (intratumoral) CDA

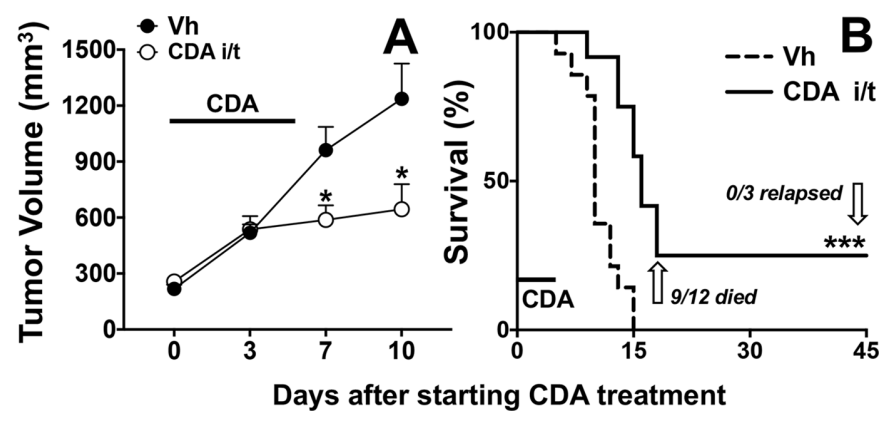

Figure 1 Cyclic diadenyl monophosphate (CDA) treatment to activate Stimulator of Interferon Genes controls Lewis lung carcinoma (LLC) tumor growth transiently. B6 mice with established $\left(250-350 \mathrm{~mm}^{3}\right)$ LLC tumors were treated with CDA (100 $\mu \mathrm{g} / \mathrm{mouse}$, intratumorally, days $0,2,6)$. Tumor volumes $(A)$ and mouse survival $(B)$ were scored until experimental end points, when all mice were examined to assess if primary tumor burdens were absent (tumor clearance), or if residual tumor tissues and/or distal metastatic tumors were present (tumor relapse). Data (mean \pm SEM) were analyzed using two-way analysis of variance with Bonferroni's multiple comparisons test for each time point $(A)$ or log-rank test $(B), n=12 .{ }^{*} P<0.05,{ }^{* * *} p<0.001$. Vh, vehicle.

treatment $(100 \mu \mathrm{g}$ on days $0,2,6)$. CDA treatment did not slow tumor growth immediately (day 3 ) but after the final CDA treatment tumor growth was reduced significantly (figure 1A). However, tumor control was transient as growth resumed in most mice, and most CDA-treated mice $(75 \%)$ had to be sacrificed by 15 days after CDA treatment was initiated due to excessive tumor growth, extending survival by only $\sim 5$ days more than control mice treated with vehicle (figure 1B). CDA-treated mice that did not succumb to tumor growth $(\sim 25 \%)$ were challenged with a second bolus of LLC cells on the contralateral flank when primary lesions had healed, 60 days after CDA treatment was initiated. Secondary LLC tumors grew at the same rates as primary tumors in all mice (table 1, group B), indicating that direct CDA treatment did not incite stable protective antitumor immunity, even though primary tumors were eliminated by CDA monotherapy.

In a previous study, CDA treatment to activate STING protected $50 \%$ of mice implanted with immunogenic B16 melanomas until experimental end points and surviving mice all acquired stable protective antitumor immunity. ${ }^{11}$ As CDA treatment was initiated when B16 tumors were relatively small $\left(\sim 100 \mathrm{~mm}^{3}\right)$ in this prior study, we next tested if CDA treatment was more effective in mice with smaller LLC tumors. Mice were treated with CDA as before, except that treatment was initiated when LLC tumors were first detected $\left(90-120 \mathrm{~mm}^{3}\right), 4-5$ days earlier than in our initial study on mice with more established tumors. Earlier CDA treatment prevented progression of small LLC tumors, leading to rapid tumor regression and uniform survival (online supplementary figure S1A,B). In addition, earlier CDA treatment induced all mice to acquire stable protective immunity to LLC challenge (table 1, group G). Direct CDA treatment was necessary 
Table 1 CDA reinforces immune regulation to attenuate antitumor responses

\begin{tabular}{|c|c|c|c|c|c|c|c|c|c|c|}
\hline \multirow[b]{3}{*}{ Group } & \multirow[b]{3}{*}{ CDA } & \multirow{2}{*}{\multicolumn{3}{|c|}{$\begin{array}{l}\text { Co-treatment } \\
\text { (with CDA) }\end{array}$}} & \multirow{2}{*}{\multicolumn{3}{|c|}{ Induced ICP pathways }} & \multicolumn{3}{|c|}{ Outcomes } \\
\hline & & & & & & & & \multirow[t]{2}{*}{ Survival* $^{*}$} & \multirow[t]{2}{*}{ Relapse† } & \multirow[t]{2}{*}{ Immunity } \\
\hline & & $\alpha P D-1$ & IDOi & coX2i & PD-L1 & IDO & cox2 & & & \\
\hline B & + & & & & + & + & + & $11 / 47$ & $0 / 11$ & $0 / 11 \ddagger$ \\
\hline C & + & + & & & & + & + & $15 / 15$ & $15 / 15$ & - \\
\hline$E$ & + & + & BMS & & & & & $8 / 9$ & $8 / 8$ & - \\
\hline $\begin{array}{l}\mathrm{F} 1 \\
\mathrm{~F} 2\end{array}$ & $\begin{array}{l}+ \\
+\end{array}$ & & & $\begin{array}{l}+ \\
+\end{array}$ & $\begin{array}{l}+ \\
+\end{array}$ & - & & $\begin{array}{l}15 / 15 \\
8 / 9\end{array}$ & $\begin{array}{l}0 / 15 \\
0 / 8\end{array}$ & $\begin{array}{l}\text { 15/15‡ } \\
8 / 8 \S\end{array}$ \\
\hline
\end{tabular}

Numbers represent total number of mice from combined experiments.

${ }^{*}$ No. of mice surviving to end point ( $>60$ days).

$\dagger$ No. of mice with tumors at end points (day 60 for groups A-F1, day 30 for group F2).

$\ddagger$ No. of mice resistant to LLC re-challenge at day 60 .

$\S N$. of mice resistant to primary and distal tumor growth (abscopal effects).

CDA, cyclic diadenyl monophosphate; COX2i, cyclooxygenase-2 inhibitor; ICP, immune checkpoint; IDOi, indoleamine 2,3 dioxygenase inhibitor; LLC, Lewis lung carcinoma; PD-1, programmed death-1; PD-L1, programmed death ligand-1.

to control LLC tumor growth as intravenous CDA treatment when tumors first appeared had little impact on tumor growth and had no significant effect on survival (online supplementary figure S1C,D). Direct CDA treatment did not protect STING-deficient (STING-KO) mice with small LLC tumors (online supplementary figure S2), confirming that therapeutic responses were STINGdependent and revealing that STING expressed by LLC tumor cells was not the relevant therapeutic target of CDA.

\section{Direct CDA treatment stimulates multiple immune regulatory pathways in the TME}

As resistance to the antitumor effects of CDA developed rapidly as tumors became more established, we assessed if CDA treatment induced immune regulatory pathways that commonly contribute to ICPs and impede antitumor immunity, as potential causes of therapy resistance. Cells expressing programmed death ligand-1 (PD-L1) induce T cells expressing PD-1 to become functionally exhausted and/or undergo apoptosis. Like many patients with cancer, mice with LLC tumors are poorly responsive to blockade of PD-1/PD-L1 signaling. ${ }^{2-5}$ Direct CDA treatment induced rapid and significant increase in PD-L1 gene transcription in tumor lesions (figure 2A) and TDLNs (figure 2B), and elevated PD-L1 expression was sustained for at least 6 hours in both TME sites. COX2 expression increases in the TME during LLC tumor growth and COX2 inhibitors slow LLC tumor growth. ${ }^{13} 14$ Direct CDA treatment potentiated COX2 gene transcription rapidly in tumor lesions and TDLNs, and elevated COX2 transcription persisted for over 6 hours in both TME sites (figure 2C,D).
Next, we tested if direct CDA treatment induced IDO enzyme activity in tumor lesions and TDLNs by assessing production of kynurenine (Kyn), a tryptophan catabolite made by cells expressing IDO, in TME tissues from
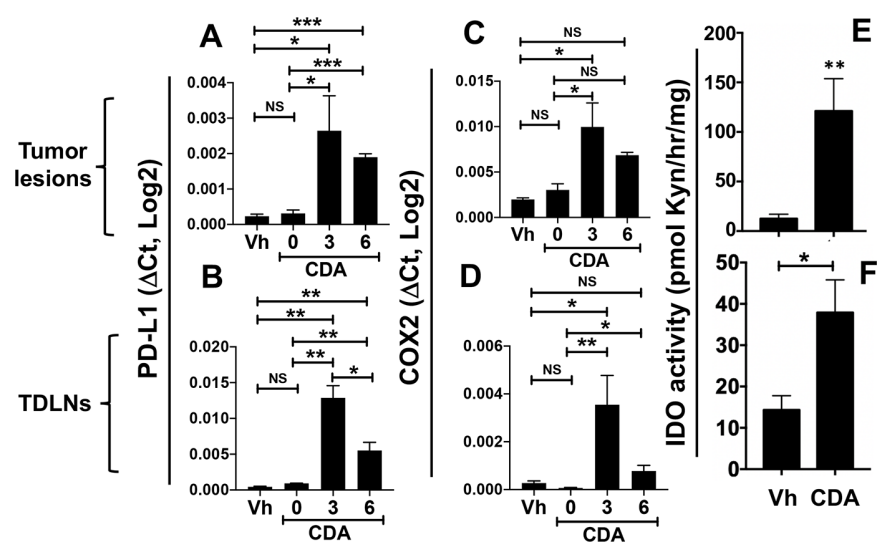

Figure 2 Direct cyclic diadenyl monophosphate (CDA) treatment enhances immune regulatory pathways in the tumor microenvironment (TME). (A-D) Mice with established Lewis lung carcinoma (LLC) tumors $\left(250-350 \mathrm{~mm}^{3}\right.$ ) were treated with CDA twice, sacrificed at the times indicated after the final CDA treatment ( 0 hour), and programmed death ligand-1 (PD-L1) and cyclooxygenase-2 (COX2) gene transcription in tumor lesions (A, C) and tumor-draining lymph nodes (TDLNs) (B, D) was assessed by qRT-PCR. Vehicle (Vh)-treated mice were sacrificed 3 hours after treatment. (E, F) Mice with established LLC tumors were treated with CDA twice and 24 hours later tumor lesion (E) and TDLN (F) tissues were homogenized to assess indoleamine 2,3 dioxygenase (IDO) activity (see 'Methods' section). Data (mean \pm SEM) were analyzed using Mann-Whitney $U$ tests, $n=3-6$. ${ }^{*} P<0.05$, ${ }^{\star *} p<0.01,{ }^{* \star *} p<0.001$. NS, not significant. 


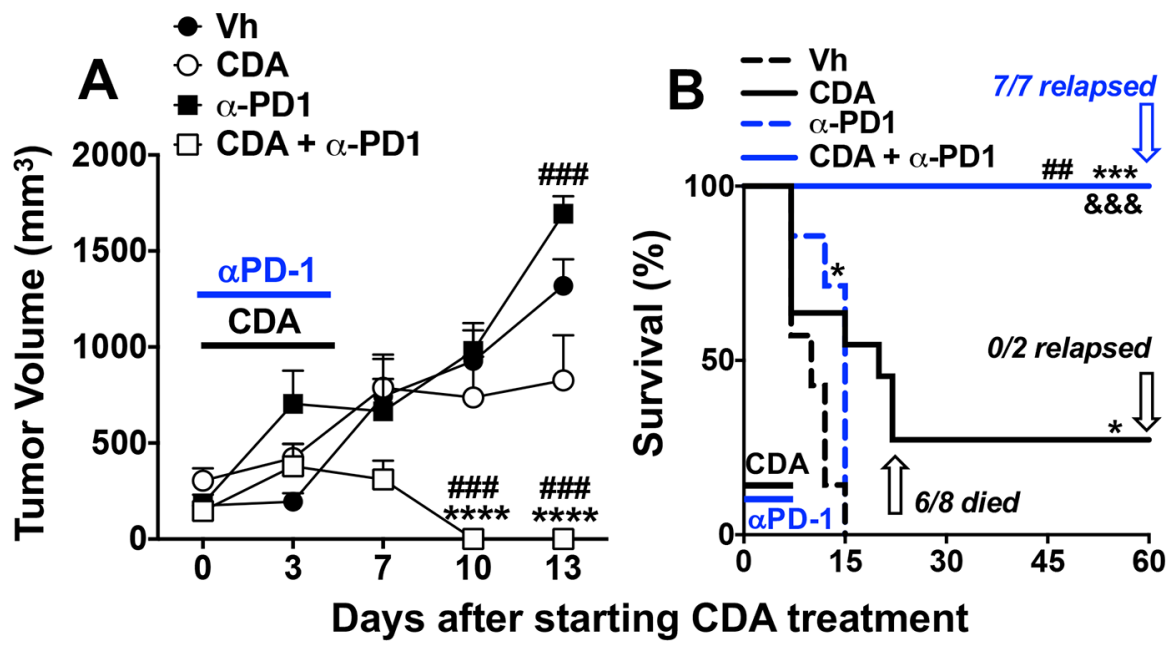

Figure 3 Programmed death-1 (PD-1) blockade enhances antitumor responses to cyclic diadenyl monophosphate (CDA). (A, B) B6 mice with established Lewis lung carcinoma (LLC) tumors were treated with CDA with or without anti-PD-1 monoclonal antibodies (mAbs) to disrupt immune checkpoints (ICPs) (see 'Methods' section). Tumor volumes (A) and mouse survival (B) were assessed until experimental end points (day 60). At end points mice were examined to assess tumor clearance or relapse. Data (mean \pm SEM) were analyzed using two-way analysis of variance with Bonferroni's multiple comparisons test for each time

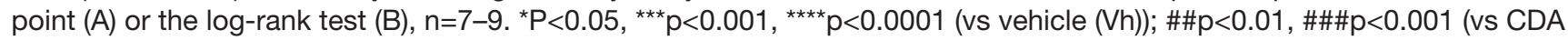
monotherapy); \&\&\& $\mathrm{p}<0.001$ (vs anti-PD1 monotherapy).

mice with LLC tumors. LLC tumor cells do not express IDO but IDO activity induced in TDLN DCs via STING/ IFN-I signaling during LLC tumor growth suppresses antitumor immunity to promote optimal LLC tumor growth. ${ }^{12}$ STING agonists also induced DCs to express IDO, an attribute we exploited to attenuate autoimmune disease in mouse models of rheumatoid arthritis, multiple sclerosis and type I diabetes. ${ }^{15-19}$ Direct CDA treatment potentiated IDO activity rapidly and significantly in tumor lesions and TDLNs, relative to levels induced by LLC growth (figure 2E,F), Thus, intratumoral STING activation induced rapid increase in local expression of PD-L1, COX2 and IDO, which may attenuate the antitumor attributes of CDA.

\section{PD-1 blockade enhances antitumor responses to CDA}

To test if CDA-induced PD-L1 expression reinforces ICP potency mice were co-treated with CDA and anti-PD-1 monoclonal antibodies (mAbs) to block PD-L1/PD-1 interactions that inactivate effector $\mathrm{T}$ cells. Consistent with LLC tumors being refractory to PD-1 blockade, anti-PD-1 mAb monotherapy (150 $\mu \mathrm{g} /$ mouse, intraperitoneal, $15 \mathrm{~min}$ before each CDA injection) did not slow LLC tumor growth (figure 3A), and had minimal survival impact (figure 3B). As before (figure 1), direct CDA monotherapy did not prevent tumor growth in most mice $(75 \%)$. Combining direct CDA treatment with PD-1 blockade led to rapid and uniform tumor regression and uniform survival until experimental end points (figure 3). No overt evidence of tumor relapse was observed in any mice co-treated with CDA and anti-PD-1 mAbs but some mice became moribund at day 60 prompting their sacrifice. On examination, all mice that became moribund had invasive and dispersed tumors originating at primary lesions and expanding into the peritoneal cavity (ie, they were not visible externally), and some mice also had distal (metastatic) tumors growing in lungs and/or liver (table 1, group C). These observations prompted sacrifice of remaining mice in this treatment group and on inspection all mice had invasive internal tumors and some had metastatic tumors. Thus, combining CDA and PD-1 blockade enhanced tumor control and survival but did not eliminate primary tumor burdens, indicating that induced antitumor immunity was transient and unstable.

\section{IDO inhibition enhances antitumor responses to CDA}

Next, we tested if three different proprietary IDO inhibitor (IDOi) drugs with distinct pharmacological characteristics enhanced therapeutic responses to direct CDA treatment in the LLC tumor model. Indoximod (1-methyl-D-tryptophan (1MT)), navoximod (NLG-919) and lindrostat (BMS-986205) have all been tested in patients with cancer as monotherapies, or in combination with other anticancer treatments, with varying effects on clinical outcomes. ${ }^{10}$ Pharmacologically, lindrostat is the most effective IDO inhibitor, although many factors may impact the antitumor attributes of IDOi drugs. ${ }^{10}$ Mice with established LLC tumors were given CDA or IDOi monotherapy, or co-treated with CDA and an IDOi drug. As before, direct CDA monotherapy prevented tumor growth in only $20 \%-30 \%$ of mice (figure 4 ). Monotherapies with 1MT (2 mg/mL in drinking water days 1-10) or lindrostat (2 $\mathrm{mg} / \mathrm{kg}$, daily gavage, days $0-10)$ had no significant effects on tumor growth (figure 4A,E) or survival (figure 4B,F), relative to vehicle-treated mice. Navoximod (intraperitoneal, $5 \mathrm{mg} / \mathrm{kg}$, daily from days 1-10) monotherapy slowed tumor growth and increased survival by $5-6$ days (figure $4 \mathrm{C}, \mathrm{D}$ ).

Co-treatment with $1 \mathrm{MT}$ did not enhance CDA-mediated tumor control or increase survival significantly, relative to 

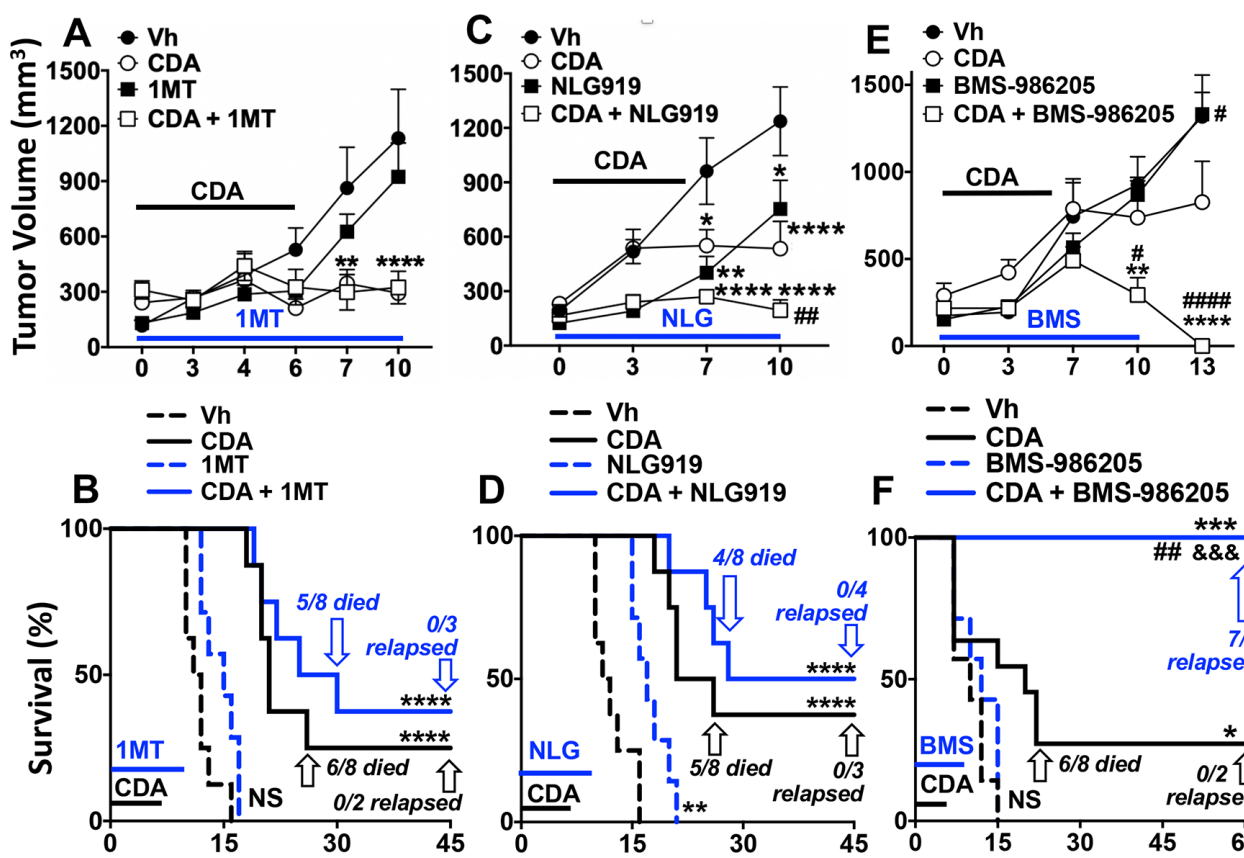

D CDA + NLG919
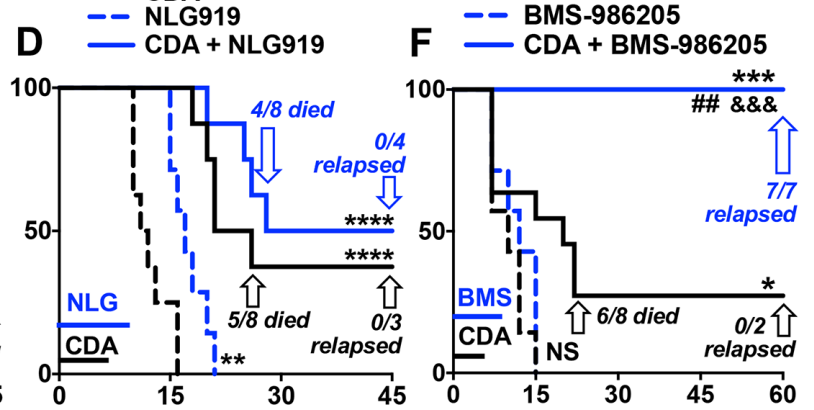

Days after starting CDA treatment

Figure 4 Indoleamine 2,3 dioxygenase (IDO) inhibition enhances antitumor responses to cyclic diadenyl monophosphate (CDA). (A-F) B6 mice with established Lewis lung carcinoma (LLC) tumors were treated with CDA with or without IDO inhibitors, $1 \mathrm{MT}$ (A, B), NLG-919 (C, D) and BMS-986205 (E, F). Tumor volumes (upper panels) and survival (lower panels) were assessed until experimental endpoints. At end points mice were examined to assess tumor clearance or relapse. Data (mean $\pm S E M)$ were analyzed using two-way analysis of variance with Bonferroni's multiple comparisons test for each time point (A, C, E) or log-rank test $(B, D, F), n=7-9$. ${ }^{*} P<0.05,{ }^{* *} p<0.01,{ }^{* \star *} p<0.0001$ (vs vehicle $\left.(V h)\right) ; \# p<0.05, \# \# p<0.01, \# \# \# \#<<0.0001$ (vs $C D A$ monotherapy); \&\&\& $\mathrm{p}<0.001$ (vs BMS-986205 monotherapy).

outcomes with CDA monotherapy (figure 4A,B). Navoximod synergized with CDA to further reduce tumor growth but did not increase survival significantly, relative to CDA monotherapy (figure 4C,D). No residual primary or metastatic tumors were found in mice surviving to experimental end points after co-treatments with CDA and $1 \mathrm{MT}$ or navoximod (figure $4 \mathrm{~B}$ and $\mathrm{D}$, table 1 , groups D1, D2), as in mice given CDA monotherapy. In contrast, lindrostat co-treatment enhanced antitumor responses to CDA significantly, promoting rapid tumor regression and uniform survival to experimental end points (figure 4E,F). Nevertheless, on inspection at experimental end points (day 60) all mice in this treatment group had invasive tumors originating at primary lesions growing into the peritoneal cavity, and some mice also had metastatic tumors in lungs and liver (table 1, group D3). Thus, as in mice co-treated with CDA and PD-1 blockade, CDA and lindrostat co-treatment induced rapid tumor regression but did not eliminate primary tumor burdens, leading to tumor relapse at primary and distal sites. Collectively, our findings reveal that increased IDO activity in the TME due to STING activation attenuates antitumor responses to $\mathrm{CDA}$, and lindrostat was the only one of three proprietary IDOi drugs tested that synergized with CDA to enhance antitumor responses substantively.

DCs expressing IDO in the TME suppress effector T cells and activate regulatory CD4 T cells (Tregs) to attenuate T cell immunity via PD-1-dependent mechanisms. ${ }^{20}$
To investigate potential links between these $\mathrm{T}$ cell regulatory pathways, we assessed if PD-1 blockade or IDOi drugs impacted IDO activity and PD-L1 expression induced by CDA treatment. PD-1 blockade had no effect on CDAinduced IDO activity in tumor lesions or TDLNs (online supplementary figure S3A,B). Lindrostat (BMS) co-treatment partially reduced CDA-induced PD-L1 expression in tumor lesions but had no significant impact on CDAinduced PD-L1 expression in TDLNs (online supplementary figure S3C,D). Thus, the IDO and PD-1 pathways make non-redundant contributions that promote resistance to STING activation independently, as disrupting either pathway enhanced antitumor responses to CDA. Since co-treatments to activated STING and block PD-1 or inhibit IDO led to eventual tumor relapse, we tested if simultaneous PD-1 and IDO blockade improved tumor control. Lindrostat plus PD-1 blockade co-treatment did not enhance tumor control or increase survival, relative to outcomes in vehicle-treated mice (online supplementary figure $\mathrm{S} 3 \mathrm{E}, \mathrm{F}$ ), reflecting outcomes reported for the ECHO-301 clinical trial using epacadostat to inhibit IDO activity. ${ }^{9}$ As expected, combined lindrostat and PD-1 blockade enhanced tumor control and prolonged survival after CDA treatment without dermal tumor relapse (online supplementary figure S3E,F). However, on inspection at experimental end points all mice in this treatment group had invasive tumors originating from primary lesions growing into the peritoneal cavity, and 


\section{Dermal LLC tumors (i/d injection)}
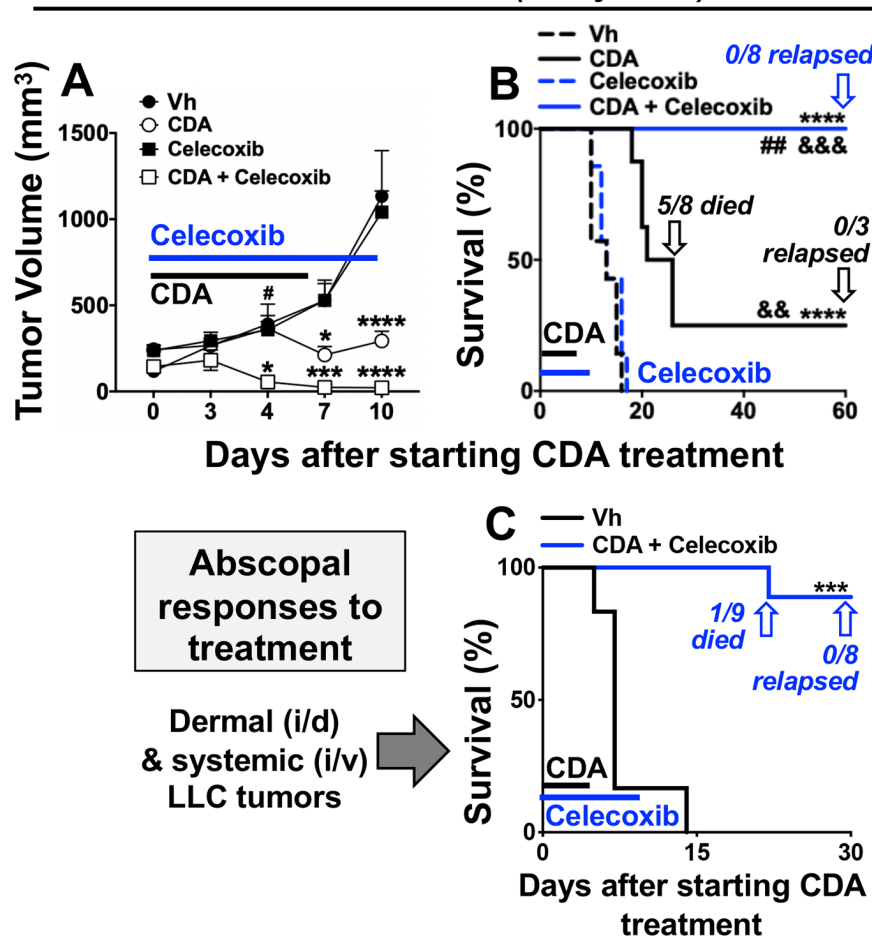

Figure 5 Celecoxib and cyclic diadenyl monophosphate (CDA) co-treatments induce protective antitumor immunity. (A, B) B6 mice with Lewis lung carcinoma (LLC) tumors were treated with CDA with or without celecoxib $(60 \mathrm{mg} /$ $\mathrm{kg}$, oral gavage) and tumor volumes (A) and mouse survival (B) were assessed. At end points mice were examined to assess tumor clearance or relapse. (C) Mice were injected with LLC cells on day $0\left(2 \times 10^{5}\right.$ cells/mouse, intradermal $\left.(\mathrm{i} / \mathrm{d})\right)$ and again on day $5\left(5 \times 10^{5}\right.$ cells/mouse, intravenous $\left.(\mathrm{i} / \mathrm{v})\right)$ and were treated as indicated. Data (mean \pm SEM) were analyzed using two-way analysis of variance with Bonferroni's multiple comparisons test for each time point $(A)$ or log-rank test $(B$, C), $n=7-9$. ${ }^{*} P<0.05,{ }^{* *} p<0.01,{ }^{* * *} p<0.001,{ }^{* * *} p<0.0001$ (vs vehicle (Vh)); \#\#p<0.01 (vs CDA monotherapy). \&\&P<0.01, $\& \& \& p<0.001, \& \& \& \& p<0.0001$ (vs celecoxib monotherapy).

some mice also had metastatic tumors (table 1, group E). Thus, simultaneous disruption of the PD-1 and IDO regulatory pathways did not improve antitumor responses to CDA, as tumor relapse still occurred in all mice given such treatments.

\section{COX2 inhibition and STING activation synergize to induce protective antitumor immunity}

Next, we tested if a COX2-selective inhibitor, celecoxib, boosted antitumor responses to CDA. Mice with established LLC tumors were co-treated with CDA (dosing as before) and celecoxib (60 mg/ $\mathrm{kg}$, oral gavage, days $0-10)$. Celecoxib monotherapy had no effect on tumor growth or survival, while (as before) CDA monotherapy slowed tumor growth and improved survival outcomes but did not prevent most mice from succumbing to tumor growth (figure 5A,B). Co-treatment with celecoxib enhanced antitumor responses to CDA significantly, inciting rapid tumor regression and uniform survival without apparent tumor relapse until experimental end points (figure 5A,B). Moreover, no mice in this treatment group became moribund and all mice were challenged with LLC cells 60 days after initiating treatment to assess their immune status. No tumor growth was observed in mice surviving celecoxib and CDA treatment and no tumors were detected at primary lesions or at distal sites in other tissues on inspection 60 days after secondary LLC challenge (table 1, group F1). Thus, co-treatment with celecoxib and CDA induced durable protective antitumor immunity that eliminated primary tumor burdens and prevented tumor metastasis.

Since celecoxib and CDA co-treatments induced potent and robust antitumor immunity, we tested if this drug combination induced abscopal immunity that prevented growth of distal tumors not directly exposed to CDA. Accordingly, mice were inoculated to seed dermal LLC tumors (day 0 ) and a second bolus of LLC cells was administered 5 days later via intravenous injection to seed distal tumors in other tissues, including lungs. Celecoxib and CDA co-treatments were initiated when dermal tumors were established (as before) and 8/9 mice survived until experimental end points on day 30 (figure $5 \mathrm{C}$ ). On inspection, no tumor growth was evident in lungs or other tissues of the eight surviving mice (table 1, group F2), indicating that celecoxib and CDA co-treatment stimulated abscopal immunity that targeted distal tumors not exposed directly to $\mathrm{CDA}$, as well as primary dermal tumors exposed to CDA. Collectively, our findings reveal that CDA-induced COX2 poses a major barrier to eliciting protective antitumor responses after direct STING activation in the TME, as inhibiting COX2 led to fully protective antitumor responses to $\mathrm{CDA}$ that prevented growth of primary tumors and incited abscopal immunity to prevent growth of distal tumors not directly exposed to $\mathrm{CDA}$.

To investigate how celecoxib impacts immune responses following STING activation, we analyzed expression of immune response genes in tumor lesions from CDA-treated mice with established LLC tumors. As expected, CDA treatment stimulated local transcription of genes encoding pro-inflammatory cytokines, IFN- $\beta$, IFN- $\gamma$, tumor necrosis factor (TNF)- $\alpha$ and interleukin (IL)-6 (figure 6A-D). Celecoxib co-treatment potentiated CDA-induced cytokine gene transcription, eliciting 10 -fold to 40 -fold increase in IFN- $\beta$, TNF- $\alpha$ and IL- 6 transcription, relative to CDA monotherapy, while celecoxib monotherapy had no effect on basal cytokine gene transcription levels. Celecoxib also potentiated CDA-induced increase in matrix metallopeptidase 3 and 13 (MMP3, MMP13) transcription (figure 6E,F). Moreover, granzyme B (GZMB) transcription was elevated in tumor lesions given CDA and celecoxib co-treatments but was not elevated after CDA monotherapy (figure 6G), suggesting that activated effector T cells expressing GZMB accumulated in tumor lesions only if celecoxib was combined with late CDA treatment.

In summary, inhibiting COX2 activity was more effective in unleashing the antitumor potential of CDA than 

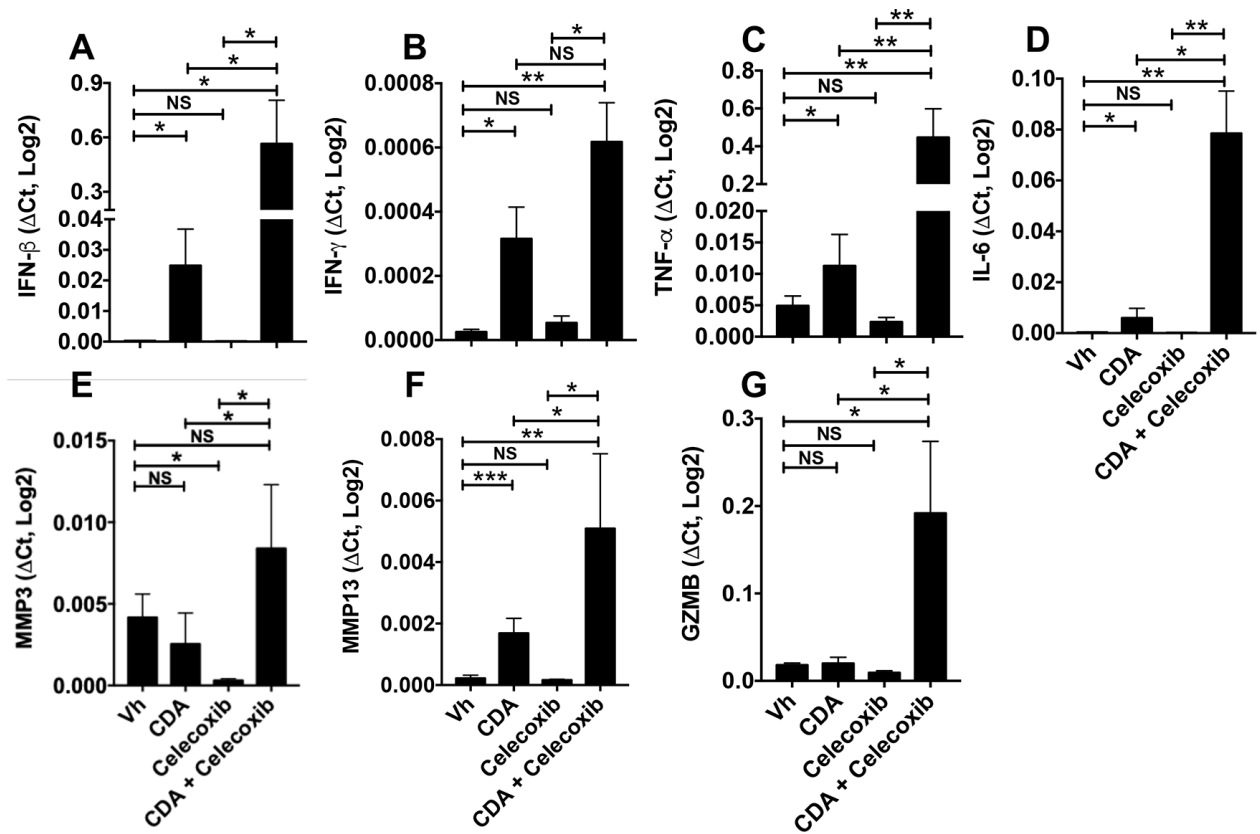

Figure 6 Celecoxib potentiates cyclic diadenyl monophosphate (CDA)-induced antitumor immunity. PCR analysis of gene expression in tumor lesions from mice bearing dermal Lewis lung carcinoma (LLC) tumors 3 hours after the second CDA treatment (as in figure 5). Data (mean $\pm S E M$ ) were analyzed using Mann-Whitney $U$ tests, $n=4-7$. ${ }^{*} P<0.05,{ }^{* \star} p<0.01,{ }^{* \star *} p<0.001$. GZMB, granzyme B; IFN, interferon; IL, interleukin; MMP, matrix metalloproteinase; NS, not significant; TNF, tumor necrosis factor; Vh, vehicle.

simultaneous disruption of the PD-1 and IDO pathways that commonly contribute to ICPs and promote therapy resistance in many patients with cancer and mouse tumor models. To assess if celecoxib impacts other STINGresponsive immune regulatory pathways, we assessed PD-L1 transcription and IDO activity in tumor lesions and TDLNs after CDA treatment. Celecoxib co-treatment completely blocked CDA-induced IDO activity in tumor lesions and TDLNs (figure 7A,B), as IDO activity levels were comparable in untreated mice with tumors and in mice co-treated with celecoxib and CDA. Celecoxib did not block CDA-induced PD-L1 transcription after STING activation (figure 7C,D). Lindrostat treatment to inhibit IDO (figure 7E,F) and PD-1 blockade (figure 7G,H) had no significant impact on CDA-induced COX2 transcription in tumor lesions or TDLNs. Thus, the superior efficacy of celecoxib, relative to IDO inhibition and PD-1 blockade, may in part be due to effective disruption of the induced IDO pathway, although celecoxib may also disrupt other regulatory pathways responsive to CDA since lindrostat was not as effective as celecoxib in unleashing the full antitumor effects of CDA.

\section{DISCUSSION}

In this study, we show that robust resistance to the antitumor effects of STING is an adaptive feature of the TME in mice with established LLC tumors. STING activation ${ }^{21}$ and PD-1 blockade to disrupt ICPs ${ }^{1}$ are complementary strategies to stimulate innate immune cells or reactivate dysfunctional tumor-specific $\mathrm{T}$ cells, respectively. Both strategies were discovered and validated in mouse tumor models, prompting clinical trials. Clinical trials to test STING activator drugs are ongoing, ${ }^{11}$ although a provisional report from an active phase I trial (NCT03010176) cited no objective clinical responses to direct (intratumoral) administration of a proprietary STING activator (MK-1454) in patients with advanced solid tumors or lymphomas. ${ }^{22}$ Thus, clinical responses to STING activators may not recapitulate the high incidence of robust antitumor responses to CDA therapy observed in some mouse tumor models. ${ }^{11}$ Likewise, the high incidence of effective antitumor responses to PD-1 blockade observed in some mouse tumor models does not reflect clinical outcomes, as $>50 \%$ of patients with melanoma and $>80 \%$ of patients with NSCLC, respectively, are refractory to PD-1 blockade monotherapy. ${ }^{13}$ Disparities in the incidence of effective antitumor responses in mouse models and patients with cancer undermine the utility of mouse models as predictors of clinical responses, and prompt the need to identify models that better reflect clinical outcomes.

We selected the LLC model for this study because LLC tumors depend on intrinsic STING signaling for optimal growth because STING/IFN-I signaling elevated IDO activity in TDLN DCs that suppress antitumor immunity. ${ }^{123}$ In contrast, immunogenic B16 tumors and LLC tumors expressing neo-antigens do not depend on STING signaling for optimal growth. ${ }^{12}$ Based on these observations, we reasoned that local immune balance driving immune regulatory responses in the parental LLC model would attenuate the antitumor effects of direct STING agonist (CDA) treatment. Our findings show that adaptive resistance to direct CDA treatment is indeed a major 
Tumor
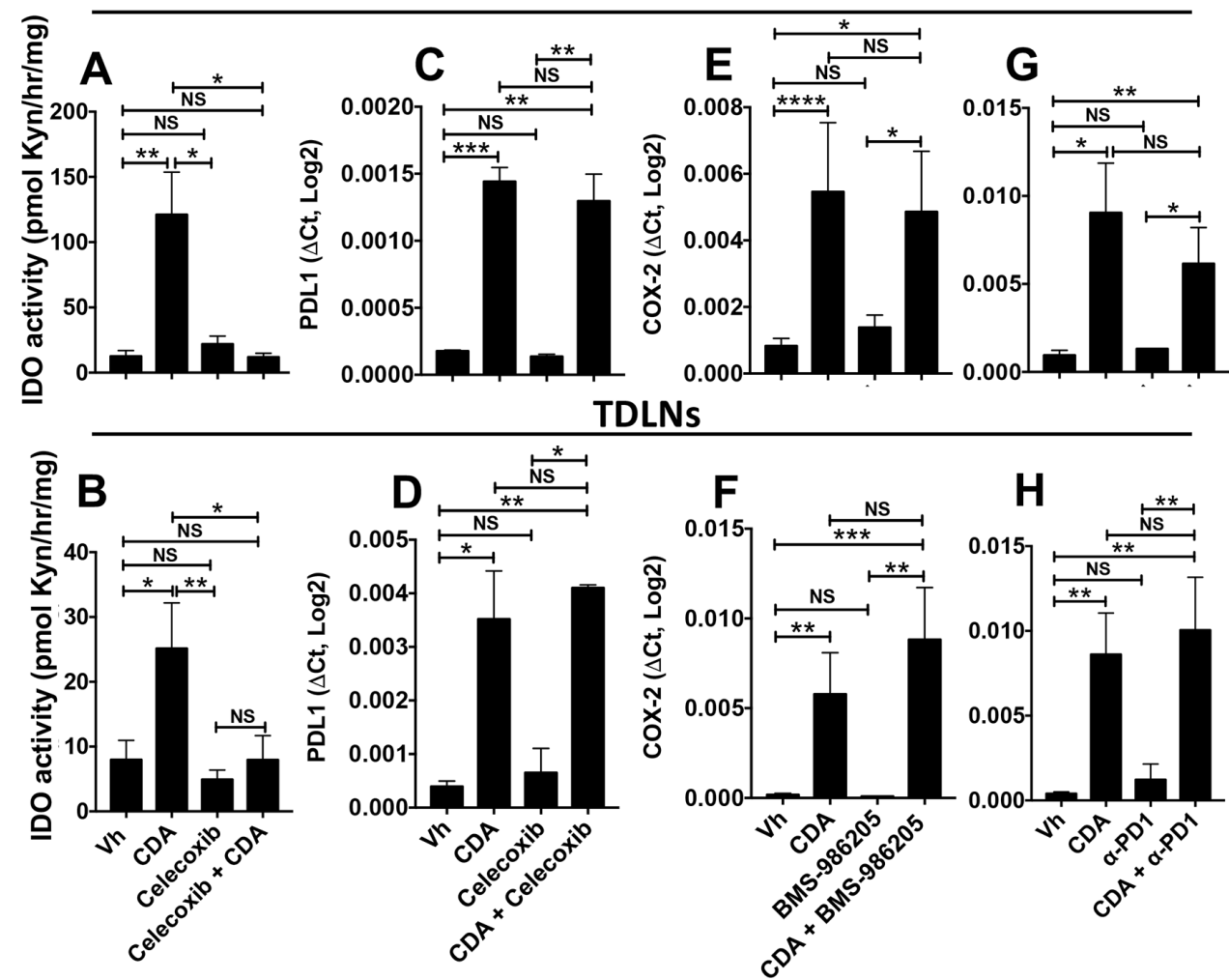

Figure 7 Celecoxib blocks indoleamine 2,3 dioxygenase (IDO) but not programmed death ligand-1 (PD-L1) induction and programmed death-1 (PD-1) or IDO blockade does not prevent cyclooxygenase-2 (COX2) induction after cyclic diadenyl monophosphate (CDA) treatment. (A-H) B6 mice bearing Lewis lung carcinoma (LLC) tumors were treated with CDA and celecoxib (A-D), CDA and BMS-986205 (E, F) or CDA and anti-PD1 monoclonal antibodies (mAbs) (G, H). Tumor lesions and tumor-draining lymph nodes (TDLNs) were harvested 24 hours (A-B) or 3 hours (C-H) after the second administration of CDA. IDO activity (A, B), PD-L1 (C, D) or COX2 gene expression $(E-H)$ was assessed. Data are mean \pm SEM. Data were analyzed using Mann-Whitney U tests, $n=4-8 .{ }^{*} P<0.05,{ }^{* *} p<0.01,{ }^{* * *} p<0.001,{ }^{* \star * *} p<0.0001$. NS, not significant; Vh, vehicle.

barrier to inciting effective antitumor responses in mice with established LLC tumors $\left(>250 \mathrm{~mm}^{3}\right)$. Resistance to CDA therapy was not an intrinsic attribute of LLC tumors, as initiating CDA monotherapy when small LLC tumors were first detected $\left(\sim 100 \mathrm{~mm}^{3}\right)$ led to uniformly protective responses against primary tumors and secondary LLC challenge, indicating that mice acquired stable antitumor immunity. Therapy resistance in mice with established LLC tumors was mediated by TME pathways that rapidly increased in potency following CDA treatment. Independent co-treatments to disrupt three distinct immune regulatory pathways increased tumor control and enhanced survival significantly following CDA treatment. Thus, adaptive resistance to CDA therapy increased as LLC tumors matured, reflecting higher resistance to immunotherapy in patients diagnosed with advanced cancers.

While PD-1 blockade monotherapy did not promote antitumor responses, PD-1 blockade synergized with CDA to reduce therapy resistance and promote uniform survival. However, surviving mice did not clear primary tumor burdens, as all mice harbored invasive residual tumors emanating from primary tumor lesions, and some mice had metastatic tumors. Two proprietary IDO inhibitor drugs with distinct pharmacological characteristics, ${ }^{10}$ 1MT and navoximod (NLG-919), had little or no antitumor effects as monotherapies, and did not improve outcomes following CDA treatment. A third proprietary IDO inhibitor drug, lindrostat (BMS-986205), with a superior pharmacological profile, reduced resistance to CDA therapy significantly, leading to uniform survival. However, as for co-treatments with CDA and PD-1 blockade, uniform tumor relapse manifested in all surviving mice co-treated with CDA and lindrostat. These outcomes reflect observations of patient resistance to PD-1 blockade and IDO inhibitor therapy in NSCLC and other cancers, ${ }^{24-26}$ and testify to the key roles of the PD-1 and IDO pathways in promoting resistance to CDA therapy. Disrupting either pathway led to significant survival benefits, indicating that these pathways were not redundant but simultaneous disruption of both pathways did not further improve CDA-induced antitumor responses. Residual or resurgent activity of PD-1 or IDO pathways, or other immune regulatory pathways following CDA therapy may explain why primary tumor burdens were not eliminated.

Metabolic adaptations, including elevated IDO activity, emerged as correlative markers of poor survival of patients with cancer with advanced melanoma and renal cell carcinoma following nivolumab treatments to block PD-1 interactions. ${ }^{6}$ Elevated serum IDO activity also associated strongly with poor survival of patients with NSCLC 
following $\mathrm{RT} / \mathrm{CT}^{78}{ }^{8}$ IDO-mediated resistance to ICP blockade was also described in mouse tumor models. ${ }^{27} 28$ Nevertheless, several IDO inhibitor drugs tested in clinical trials did not lead to significant survival benefit for patients with cancer, even when the proprietary IDO inhibitor epacadostat was administered in combination with pembrolizumab to block PD-1 interactions in the ECHO-301 phase III clinical trial. ${ }^{9}$ Our findings in the LLC model confirm that IDO activity dampens the antitumor attributes of direct STING activation, and testify to the technical difficulty of sustaining pharmacological IDO inhibition to overcome therapy resistance in the TME.

The COX2-specific inhibitor celecoxib was more effective in disrupting adaptive resistance to CDA therapy than PD-1 blockade or lindrostat co-treatments, even if these co-treatments were combined. Mice co-treated with CDA and celecoxib survived uniformly and acquired systemic antitumor immunity that protected mice from growth of distal LLC tumors not exposed directly to CDA (abscopal effects) and acquired long-term (memory) immunity that prevented growth of LLC tumors after secondary challenge. These outcomes revealed that the COX2 pathway is a major factor contributing to therapy resistance, and that inhibiting COX2 activity restored the full range of antitumor responses to CDA monotherapy observed in mice with smaller B16 tumors ${ }^{11}$ and LLC (this study) tumors. This finding is consistent with previous reports that COX2, and the ensuing prostaglandin cascade, promote carcinogenesis and suppress antitumor immunity, potentially by attenuating IFN signaling in the TME. ${ }^{2-31}$ Celecoxib and the non-specific COX1/2 inhibitor aspirin reduced cancer risk but did not slow growth of immunogenic tumors in mice. ${ }^{29} 30$ Aspirin boosted antitumor responses to PD-1 blockade but synergistic effects were modest, even though treatment was initiated only 3 days after tumor engraftment. ${ }^{30} \mathrm{COX} 2$ inhibitors also slowed LLC tumor growth, ${ }^{13} 14$ although effects were modest, despite administering drug continuously starting 4 days after tumor engraftment. ${ }^{13}$ In the current study, celecoxib monotherapy had no survival benefit in mice with established LLC tumors, despite its potent synergistic effects when combined with CDA treatment. Celecoxib potentiated CDA-induced expression of genes associated with inflammation and immunity, including cytokines, extracellular matrix remodeling enzymes and GZMB expressed by effector T cells. Since STING-deficient mice bearing STING-sufficient LLC tumor cells were refractory to the antitumor effects of CDA, tumor-associated cells, not tumor cells, are the source of STING-induced IFN- $\beta$ that drives antitumor responses. The ability of celecoxib to boost inflammatory and immune responses appears paradoxical, as the anti-inflammatory attributes of COX2 inhibitors are well known. A potential reason why celecoxib enhanced inflammatory and immune responses to CDA is that celecoxib blocked local increase in CDA-induced IDO activity, a potent suppressor of innate and adaptive immunity. ${ }^{15}$ This finding is consistent with previous reports that
COX2-specific inhibitors blocked IDO induction in acute myeloid leukemia cells and in mice with LLC tumors. ${ }^{142}$ However, contrasting outcomes following co-treatments with CDA and celecoxib or lindrostat imply that IDO blockade only partially explains the superior antitumor effects of celecoxib. As IFNs stimulate PD-L1 and IDO expression, it was surprising that elevated IFN production following $\mathrm{CDA} /$ celecoxib co-treatment did not upregulate these regulatory pathways. However, factors that modulate PD-L1 expression and IDO activity in distinct cell types are poorly defined. For example, inhibiting the COX2/prostaglandin pathway reduced PD-L1 expression in tumor-associated macrophages (TAMs) and myeloidderived suppressor cells in mice with MBT2 bladder carcinomas ${ }^{33}$ and induced TAMs to adopt an inflammatory M1 phenotype in mice with $\mathrm{APC}^{\mathrm{Min}}$ intestinal tumors. ${ }^{34}$

In clinical settings, the requirement for direct CDA administration into tumors may be challenging, although improved image-guided drug delivery makes this approach increasingly feasible. Some STING activators may be effective when given systemically. 5,6-Dimethylxanthenone-4-acetic acid (DMXAA), which activates murine but not human STING, blocked growth of small LLC tumors when given via the intraperitoneal route, although (as for CDA) DMXAA was less effective in controlling growth of larger LLC tumors and survival was not reported. ${ }^{35}$ Intravenous treatment with a synthetic derivative of the natural STING activator cyclic guanyl-adenyl monophosphate (cGAMP), made by the cytosolic DNA sensor cGAMP synthase, slowed dermal CT-26 adenocarcinoma growth and enhanced mouse survival. ${ }^{36}$ However, these outcomes were achieved only at the highest dose tested $(20 \mathrm{mg} / \mathrm{kg})$ and dosing was initiated at CT-26 engraftment, a clinically impractical dosing regimen. In this study, survival assessments were curtailed prematurely, as $40 \%$ of vehicle-treated mice survived until experimental end points. Another study using dimerized amidobenzimidazole (diABZI) reagents to activate STING controlled CT-26 tumor growth and promoted survival when administered intravenously, although diABZI treatment was initiated just 2 days after CT-26 engraftment. ${ }^{37}$ Thus, STING activators may have considerable potential as antitumor immune adjuvants but use of clinically unfeasible treatments and outcome measures lacking rigor make it difficult to estimate the likely efficacy of STING activators in patients.

In summary, adaptive therapy resistance is a major barrier to achieving effective antitumor responses following direct activation of STING, particularly when the TME is mature. Our findings suggest that STING agonist monotherapy may not provoke durable and abscopal antitumor immunity in settings of clinical cancer, unless treatment is given directly to relatively small tumors. Our findings in the LLC tumor model testify to the remarkable physiological plasticity of the mature TME, and identify potential strategies to reduce therapy resistance by targeting STING-responsive pathways that suppress antitumor immunity and inflammation. For these reasons, 
the LLC model offers novel insights into robust barriers that preclude successful clinical immunotherapy, as mice with established LLC tumors reflect high resistance to immunotherapy in patients diagnosed with advanced cancers.

\section{METHODS \\ Mice}

C57BL/6 (B6) mice were bred in a barrier facility at the Comparative Biology Centre, Newcastle University. STING-KO mice (fully backcrossed to $\mathrm{B} 6$ background) were described previously. ${ }^{38}$

\section{Tumor models}

LLC (ATCC) tumor cells were injected intradermally $\left(2 \times 10^{5}\right.$ cells $/$ mouse $)$ into the right flank of female B6 mice and tumor growth was monitored. Tumor sizes were calculated using the formula $\mathrm{V}=(\mathrm{d} 1 \times \mathrm{d} 2)^{3 / 2} \times(\varpi / 6)$, where $\mathrm{d} 1$ and $\mathrm{d} 2$ are perpendicular tumor diameters. Mice were treated with mixed linkage CDA ammonium salt (ML RR-S2 CDA or AKA ADU-S100; Insight Biotechnology, $100 \mu \mathrm{g} /$ mouse, intratumorally or intravenously) or vehicle (deionized water), as described previously. ${ }^{11}$ Some mice were treated with celecoxib (Sigma-Aldrich, $60 \mathrm{mg} / \mathrm{kg}$, oral gavage), 1MT (2 mg/mL, drinking water), navoximod (NLG-919, $5 \mathrm{mg} / \mathrm{kg}$, intraperitoneal), kindly provided by NewLink Genetics, BMS-986205 (2 $\mathrm{mg} / \mathrm{kg}$, oral gavage), kindly provided by Bristol-Myers Squibb, anti-mPD1 (Clone RMP1-14, BioXcell), antiIFNAR (MAR1-5A3), IgG2a isotype (clone 2A3, BioXcell) or IgG1 isotype control (MOPC-21, BioXcell) mAbs (150 $\mu \mathrm{g}$ /injection, $15 \mathrm{~min}$ before each CDA injection). Vehicles for BMS, navoximod and celecoxib were $5 \%$ dimethyl sulfoxide/10\% Tween 20/30\% polyethylene glycol/55\% phosphate-buffered saline (PBS) (added in this order). $1 \mathrm{MT}$ was dissolved in $0.1 \mathrm{M} \mathrm{NaOH}, \mathrm{pH}$ adjusted to 7.4 with $0.1 \mathrm{M} \mathrm{HCl}, 10$ tablets/1 of Hermesetas (Sodium Saccharin, Sucralose; Carrier: L-Leucine) sweetener added, and volume adjusted with water to give $2 \mathrm{mg}$ / $\mathrm{mL}$. To evaluate abscopal effects, mice were injected with $2 \times 10^{5}$ cells/mouse (intradermally) and 5 days later with $5 \times 10^{5}$ cells/mouse (intravenously).

\section{ID0 enzyme activity}

IDO activity was measured in TDLN or tumor lesions as described previously. ${ }^{16}$ In brief, tissues were homogenized in PBS, added to IDO enzyme cocktails and kynurenine generated after 2 hours was measured by high-performance liquid chromatography.

\section{Quantitative RT-PCR}

RNA was extracted using Tri reagent (Sigma-Aldrich), using the manufacturer's recommended procedure. RNA was then reverse-transcribed using a random hexamer cDNA RT kit (Clontech), and quantitative RT-PCR was performed using SsoFast EvaGreen supermix (Bio-Rad).
PCR primers (murine) were as follows $\left(5^{\prime}-3^{\prime}\right.$, forward and reverse);

B-actin: TACGGATGTCAACGTCACAC \&

AAGAGCTATGAGCTGCCTGA;

COX2: ATCATAAGCGAGGACCTGGG \&

CTGCAGGTTCTCAGGGATGT;

PD-L1: TGCGGACTACAAGCGAATCA \&

CTTCTCTTCCCACTCACGGG;

Granzyme B: GAAGCCAGGAGATGTGTGCT \&

GCACGTTTGGTCTTTGGGTC;

MMP3: CTATACGAGGGCACGAGGAG \&

CCACCCTTGAGTCAACACCT

MMP13: GGAGCCCTGATGTTTCCCAT \&

ATCAAGGGATAGGGCTGGGT;

TNF- $\alpha$ : TCGTAGCAAACCACCAAGTG \&

GGAGTAGACAAGGTACAACC

IL-6: AGACAAAGCCAAGT CCTTCAGAGA \& GCCA

CTCCTTCTGTGACTCCAGC

IFN- $\gamma:$ CCTTCTTCAGCAACAGCAAGGCG \&

CCCACCCCGAATCAGCAGCG

IFN- $\beta 1$ : GCAGCTGAATGGAAAGATCA \&

GTGGAGAGCAGTTGAGGACA

Threshold cycle $(\mathrm{Ct})$ values were set in the early linear phase of amplification; relative expression of target genes were calculated as $2 \mathrm{Ct}(\beta$-actin $)-\mathrm{Ct}($ target gene $)$.

\section{Statistical analysis}

Data were analyzed using GraphPad Prism. For tumor growth, two-way analysis of variance with Bonferroni post hoc multicomparison tests were performed. Unpaired two-tailed non-parametric Mann-Whitney $\mathrm{U}$ tests were performed for two group comparisons. Statistical significance for survival curves was evaluated using the log-rank test.

Twitter Jessica Calver @jessfcalver and Jack Minett @jack_minett

Acknowledgements The authors would like to thank the personnel of the Newcastle University Comparative Biology Center, especially Daimon Luke, Mike Snelling, Brigid Griffin and Christopher Huggins for their help and excellent care of the animals used in this study. The authors would also like to thank Nikita Lewis for help with some experiments.

Contributors ALM and LH co-managed the study and wrote the manuscript. HL designed and conducted experiments, analyzed data and helped write and critique the manuscript. LH also helped design experiments, acquire and analyze data. AC provided E0771 cells and expertise with the breast cancer model. R0, YL, CM, JC and JM conducted experiments.

Funding This study was supported by a grant to ALM from Cancer Research UK (Cancer Immunology Project A20970) and funds from the Faculty of Medical Sciences, Newcastle University.

Competing interests AM holds shares in NewLink Genetics (now Lumos Pharma) and has received licensing income from this source.

Patient consent for publication Not required.

Ethics approval All procedures in mice were approved by the Newcastle Animal Welfare and Ethical Review Board and performed under a UK Home Office license (license number P1B4042BB).

Provenance and peer review Not commissioned; externally peer reviewed.

Data availability statement Data sharing not applicable as no datasets generated and/or analyzed for this study. All data relevant to the study are included in the article or uploaded as supplementary information. No large datasets requiring public access are included in this study. All data relevant 
to this study are included in the manuscript or submitted as supplemental information.

Open access This is an open access article distributed in accordance with the Creative Commons Attribution 4.0 Unported (CC BY 4.0) license, which permits others to copy, redistribute, remix, transform and build upon this work for any purpose, provided the original work is properly cited, a link to the licence is given, and indication of whether changes were made. See https://creativecommons.org/ licenses/by/4.0/.

\section{ORCID iDs}

Jessica Calver http://orcid.org/0000-0003-3110-8631

Lei Huang http://orcid.org/0000-0002-1996-944X

Andrew L Mellor http://orcid.org/0000-0002-9553-6160

\section{REFERENCES}

1 Sharma P, Allison JP. Immune checkpoint targeting in cancer therapy: toward combination strategies with curative potential. Cell 2015;161:205-14.

2 Bullock BL, Kimball AK, Poczobutt JM, et al. Tumor-Intrinsic response to IFN $\gamma$ shapes the tumor microenvironment and anti-PD-1 response in NSCLC. Life Sci Alliance 2019;2:e201900328.

3 Constantinidou A, Alifieris C, Trafalis DT. Targeting programmed cell death -1 (PD-1) and ligand (PD-L1): a new era in cancer active immunotherapy. Pharmacol Ther 2019;194:84-106.

4 Sharma P, Allison JP. The future of immune checkpoint therapy. Science 2015;348:56-61.

5 Li HY, McSharry M, Bullock B, et al. The tumor microenvironment regulates sensitivity of murine lung tumors to $P D-1 / P D-L 1$ antibody blockade. Cancer Immunol Res 2017;5:767-77.

6 Li H, Bullock K, Gurjao C, et al. Metabolomic adaptations and correlates of survival to immune checkpoint blockade. Nat Commun 2019;10:4346.

7 Wang W, Huang L, Jin J-Y, et al. A validation study on IDO immune biomarkers for survival prediction in non-small cell lung cancer: radiation dose fractionation effect in early-stage disease. Clin Cancer Res 2020;26:282-9.

8 Wang W, Huang L, Jin J-Y, et al. Ido immune status after chemoradiation may predict survival in lung cancer patients. Cancer Res 2018;78:809-16.

9 Muller AJ, Manfredi MG, Zakharia Y, et al. Inhibiting IDO pathways to treat cancer: lessons from the ECHO-301 trial and beyond. Semin Immunopathol 2019;41:41-8.

10 Lemos H, Huang L, Prendergast GC, et al. Immune control by amino acid catabolism during tumorigenesis and therapy. Nat Rev Cancer 2019;19:162-75.

11 Corrales L, Glickman LH, McWhirter SM, et al. Direct activation of sting in the tumor microenvironment leads to potent and systemic tumor regression and immunity. Cell Rep 2015;11:1018-30.

12 Lemos H, Mohamed E, Huang L, et al. Sting promotes the growth of tumors characterized by low antigenicity via IDO activation. Cancer Res 2016;76:2076-81.

13 Pan C, Zhang Y, Meng Q, et al. Down regulation of the expression of ELMO3 by COX2 inhibitor suppresses tumor growth and metastasis in non-small-cell lung cancer. Front Oncol 2019;9:363.

14 Lee SY, Choi HK, Lee KJ, et al. The immune tolerance of cancer is mediated by IDO that is inhibited by COX-2 inhibitors through regulatory T cells. J Immunother 2009;32:22-8.

15 Huang L, Li L, Lemos $\mathrm{H}$, et al. Cutting edge: DNA sensing via the sting adaptor in myeloid dendritic cells induces potent tolerogenic responses. J Immunol 2013;191:3509-13.

16 Huang L, Lemos HP, Li L, et al. Engineering DNA nanoparticles as immunomodulatory reagents that activate regulatory $T$ cells. $J$ Immunol 2012;188:4913-20.
17 Lemos H, Huang L, Chandler PR, et al. Activation of the sting adaptor attenuates experimental autoimmune encephalitis. $J$ Immunol 2014;192:5571-8.

18 Lemos H, Mohamed E, Huang L, et al. Stimulator of interferon genes agonists attenuate type I diabetes progression in NOD mice. Immunology 2019;158:353-61.

19 Lemos H, Mohamed E, Ou R, et al. Co-Treatments to boost IDO activity and inhibit production of downstream catabolites induce durable suppression of experimental autoimmune encephalomyelitis. Front Immunol 2020;11:1256.

20 Munn DH, Mellor AL. Ido in the tumor microenvironment: inflammation, counter-regulation, and tolerance. Trends Immunol 2016;37:193-207.

21 Woo S-R, Corrales L, Gajewski TF. Innate immune recognition of cancer. Annu Rev Immunol 2015;33:445-74.

22 Harrington KJ, Brody J, Ingham M, et al. Preliminary results of the first-in-human (FIH) study of MK-1454, an agonist of stimulator of interferon genes (STING), as monotherapy or in combination with pembro. EMSO 2018 Conference 2018.

23 Friberg $M$, Jennings $R$, Alsarraj M, et al. Indoleamine 2,3-dioxygenase contributes to tumor cell evasion of $\mathrm{T}$ cell-mediated rejection. Int $J$ Cancer 2002;101:151-5.

24 Botticelli A, Cerbelli B, Lionetto L, et al. Can IDO activity predict primary resistance to anti-PD-1 treatment in NSCLC? J Trans/ Med 2018;16:219.

25 Toulmonde M, Penel N, Adam J, et al. Use of PD-1 targeting, macrophage infiltration, and IDO pathway activation in sarcomas: a phase 2 clinical trial. JAMA Oncol 2018;4:93-7.

26 Companies scaling back IDO1 inhibitor trials. Cancer Discov 2018;8:OF5.

27 Holmgaard RB, Zamarin D, Li Y, et al. Tumor-Expressed IDO recruits and activates MDSCs in a Treg-Dependent manner. Cell Rep 2015;13:412-24.

28 Holmgaard RB, Zamarin D, Munn DH, et al. Indoleamine 2,3-dioxygenase is a critical resistance mechanism in antitumor $\mathrm{T}$ cell immunotherapy targeting CTLA-4. J Exp Med 2013;210:1389-402.

29 Harris RE. Cyclooxygenase-2 (COX-2) and the inflammogenesis of cancer. Subcell Biochem 2007;42:93-126.

30 Zelenay S, van der Veen AG, Böttcher JP, et al. CyclooxygenaseDependent tumor growth through evasion of immunity. Cell 2015;162:1257-70.

31 Kobayashi K, Omori K, Murata T. Role of prostaglandins in tumor microenvironment. Cancer Metastasis Rev 2018;37:347-54.

32 lachininoto M, Nuzzolo E, Bonanno G, et al. Cyclooxygenase-2 (COX-2) inhibition constrains indoleamine 2,3-dioxygenase 1 (IDO1) activity in acute myeloid leukaemia cells. Molecules 2013;18:10132-45.

33 Prima V, Kaliberova LN, Kaliberov S, et al. COX2/mPGES1/ PGE2 pathway regulates PD-L1 expression in tumor-associated macrophages and myeloid-derived suppressor cells. Proc Natl Acad Sci U S A 2017;114:1117-22.

34 Nakanishi Y, Nakatsuji M, Seno H, et al. Cox-2 inhibition alters the phenotype of tumor-associated macrophages from $\mathrm{M} 2$ to $\mathrm{M} 1$ in Apcmin/+ mouse polyps. Carcinogenesis 2011;32:1333-9.

35 Jassar AS, Suzuki E, Kapoor V, et al. Activation of tumorassociated macrophages by the vascular disrupting agent 5,6-dimethylxanthenone-4-acetic acid induces an effective CD8 ${ }^{+} \mathrm{T}$ cell-mediated antitumor immune response in murine models of lung cancer and mesothelioma. Cancer Res 2005;65:11752-61.

36 Li T, Cheng H, Yuan H, et al. Antitumor activity of cGAMP via stimulation of cGAS-cGAMP-STING-IRF3 mediated innate immune response. Sci Rep 2016;6:19049.

37 Ramanjulu JM, Pesiridis GS, Yang J, et al. Design of amidobenzimidazole sting receptor agonists with systemic activity. Nature 2018;564:439-43.

38 Ishikawa H, Ma Z, Barber GN. Sting regulates intracellular DNAmediated, type I interferon-dependent innate immunity. Nature 2009;461:788-92. 